\title{
MBioTracker: Multimodal Self-Aware Bio-Monitoring Wearable System for Online Workload Detection
}

\author{
Fabio Dell'Agnola, Member, IEEE, Una Pale, Rodrigo Marino, Student, IEEE, \\ Adriana Arza, David Atienza, Fellow, IEEE
}

\begin{abstract}
Cognitive workload affects operators' performance principally in high-risk or time-demanding situations and when multitasking is required. An online cognitive workload monitoring system can provide valuable inputs to decision-making instances, such as the operator's state of mind and resulting performance. Therefore, it can allow potential adaptive support to the operator. This work presents a new design of a wearable embedded system for online cognitive workload monitoring. This new wearable system consists of, on the hardware side, a multi-channel physiological signals acquisition (respiration cycles, heart rate, skin temperature, and pulse waveform) and a low-power processing platform. Further, on the software side, our wearable embedded system includes a novel energy-aware bio-signal processing algorithm. We also use the concept of application self-awareness to enable energy-scalable embedded machine learning algorithms and methods for online subjects' cognitive workload monitoring. Our results show that this new wearable system can continuously monitor multiple bio-signals, compute their key features, and provide reliable detection of high and low cognitive workload levels with a time resolution of 1 minute and a battery lifetime of $14.58 \mathrm{~h}$ in our experimental conditions. It achieves a detection accuracy of $76.6 \%(2.6 \%$ lower than analogous offline computer-based analysis) with a sensitivity of $77.04 \%$ and a specificity of $81.75 \%$, on a simulated drone rescue mission task. Moreover, by applying our self-aware monitoring to exploit different energy-scalable modes, we can increase battery lifetime by $51.6 \%$ (up to 22.11 hours) while incurring an insignificant accuracy loss of $1.07 \%$.
\end{abstract}

Index Terms-Cognitive Workload, Embedded Systems, Online Monitoring, Physiological Signals, Wearable, Edge Processing, Self-Awareness.

\section{INTRODUCTION}

Cognitive workload (CW) affects the operator's performance, mainly in high-risk and high-demanding situations [1], [2]. Thus, by providing the operator's state and resulting performance to decisionmaking instances, we can improve the user's performance and potentially reduce hazards. Indeed, unobtrusive and real-time monitoring of cognitive workload can be used as feedback to adaptively support the operator according to his/her specific needs.

In the last years, the feasibility of workload assessment from unobtrusively measured physiological signals has been shown [3]-[6], motivating a wearable and real-time cognitive workload monitoring method. However, the physiological response of cognitive workload cannot be monitored with a single biomarker (e.g., heart rate) since

This work has been partially supported by the NCCR Robotics through the Symbiotic Drone project, by the ML-Edge Swiss National Science Foundation (NSF) Research project (GA No. 200020182009/1), and the PEDESITE Swiss NSF Sinergia project (GA No. SCRSII5 193813/1). The authors would like to acknowledge the support of Dr. Ping-Keng Jao, Dr. Ricardo Chavarriaga, and Prof. José del R. Millán on the design and implementation of the protocol for the raw EEG dataset used in this work (approval no. PB2017-00295 of CER-VD).

F. Dell'Agnola, U. Pale, A. Arza, D. Atienza are with the Embedded Systems Laboratory (ESL), École Polytechnique Fédérale de Lausanne (EPFL) Lausanne, Switzerland (email: fabio.dellagnola@alumni.epfl.ch, \{una.pale, adriana.arza, david.atienza\}@epfl.ch.

R. Marino is with the Centro de Electrónica Industrial, Universidad Politécnica de Madrid, 28006 Madrid, Spain (email: rodrigo.marino@upm.es). it provokes multiple reactions due to its multidimensional nature that also varies from one individual to another [7]-[9]. Therefore, cognitive workload monitoring requires a multi-signal measurement to capture the multidimensionality of a person's physiological response, which makes the use of wearable devices challenging.

Although previous studies have utilised wearable devices for workload detection [9]-[13], data processing is still performed either in the cloud [11], [12] or offline on a computer [9], [10] because of high data dimensionality and required computational resources. Further, data streaming is not energy-efficient for online workload detection on a daily basis and does not facilitate integration with decisionmaking instances. Furthermore, there is a lack of wearable multiparametric devices and systems that provide accurate meaningful data featuring energy efficiency and low obtrusiveness. To the best of our knowledge, there is no multi-sensor embedded system suitable for real-time cognitive workload monitoring based on edge computing.

Therefore, a clear need exists for a new wearable embedded system for multi-physiological signal acquisition and edge processing, targeting online cognitive workload monitoring. However, the design of such wearable systems and devices still imposes great challenges. A real-time multimodal monitoring system requires large amounts of signal data to be filtered, processed, transmitted and recorded. This situation affects the battery lifetime and size, and is often limited by the memory constraints of the platform used. Thus, new algorithms are needed to efficiently manage energy consumption, avoid delays, and reduce memory usage while being accurate and robust.

Recently, the concept of system self-awareness has been proposed to improve the energy efficiency of multimodal applications [13][16]. Self-awareness is the property of the system to evaluate its own performance via interaction with the environment and to take specific actions based on this feedback to maximize performance [17]. In our application, the system interacts with the environment (i.e., operator's state), understanding when a more comprehensive analysis of the physiological response to workload is required (e.g., higher dimensional feature set). In contrast, whenever the person's physiological response tends towards a normal state, a self-aware system reduces sensors number, feature set, and model complexity and, in consequence, reduces energy consumption. Therefore, it should be possible to dynamically adapt active resources count that allows confident detection performance and, at the same time, reduce energy consumption.

In this paper, we present a multimodal self-aware bio-monitoring wearable system for online workload detection (MBioTracker). Moreover, we exploit the trade-offs between model complexity and energy consumption of the system based on a confidence classification threshold, thus enabling scalable energy consumption when embedded machine learning algorithms and methods of different complexity are used.

The main contributions of this paper are the following:

- We present MBioTracker, a multimodal physiological signals acquisition and processing platform, which embeds novel energy-aware biosignal processing algorithms and methods that have been validated for online cognitive workload monitoring on the edge.

- We detect online both low- and high-cognitive workload states of 
TABLE I: State-of-the-art multimodal monitoring applications using wearable sensors.

\begin{tabular}{|c|c|c|c|c|c|c|c|}
\hline Ref. & Application & $\begin{array}{l}\text { Edge } \\
\text { Processing }\end{array}$ & $\begin{array}{l}\text { Seg. } \\
\text { Win }\end{array}$ & Methods & $\begin{array}{l}\text { Detection } \\
\text { Performance }\end{array}$ & $\begin{array}{l}\text { Battery } \\
\text { Lifetime }\end{array}$ & $\begin{array}{l}\text { Sensor Platform \& } \\
\text { (Signals) }\end{array}$ \\
\hline [13] & $\begin{array}{l}\mathrm{CW} \text { during manual } \\
\text { labor }\end{array}$ & No & $60 \mathrm{~s}$ & $\begin{array}{l}\text { RFE (13 Features), RF (3- } \\
\text { class), Self-aware (2-levels) }\end{array}$ & Gmean: $81.7 \%$ & $270 \mathrm{~mJ}^{*}$ & $\begin{array}{l}\text { Shimmer (ECG,RSP), } \\
\text { E4 (PPG, SKT, EDA) }\end{array}$ \\
\hline [12] & $\mathrm{CW}$ & No & $60 \mathrm{~s}$ & $\begin{array}{l}\text { Feat-Imp by RF (10 Features) } \\
\text { RF (2-class) }\end{array}$ & Acc: $84.1 \%$ & - & $\begin{array}{l}\text { Shimmer (ECG,RSP), } \\
\text { E4 (PPG, SKT, EDA) }\end{array}$ \\
\hline [18] & $\begin{array}{l}\text { Stress in working en- } \\
\text { vironment }\end{array}$ & No & $10 \mathrm{~min}$ & $\begin{array}{l}\text { Feat-Imp by Corr with cortisol } \\
\text { ( } 7 \text { Features) SVM (2-class) }\end{array}$ & $\begin{array}{l}\text { Acc: } 86 \% C V, \\
\text { Gmean: } 86.9 \% C V\end{array}$ & - & $\begin{array}{l}\text { MindWave (EEG), } \\
\text { BioHarness (EDA), } \\
\text { Shimmer (ECG) }\end{array}$ \\
\hline [19] & SSVEP-based BCI & $\begin{array}{l}\text { Yes (Mr. Wolf } \\
8 \text {-cores/M4) }\end{array}$ & $2 \mathrm{~s}$ & $\begin{array}{l}\text { Canonical correlations and } \\
\text { threshold technique }\end{array}$ & Acc: $82 \%$ & $\begin{array}{l}38 \mathrm{~h} \\
(65 \mathrm{mAh})\end{array}$ & $\begin{array}{l}\text { Bio-Wolf device } \\
\text { (3-EEG) }\end{array}$ \\
\hline [20] & $\begin{array}{l}\text { Health patch (Re- } \\
\text { spRate, HR, SpO2) }\end{array}$ & $\begin{array}{l}\text { Yes } \\
\text { (Cortex-M4) }\end{array}$ & - & $\begin{array}{l}\text { Hw accelerators (DAM, FFT, } \\
\text { matrix, sample rate converter) }\end{array}$ & - & $\begin{array}{l}504 \mathrm{~h} \\
(630 \mathrm{mAh})\end{array}$ & $\begin{array}{llr}\text { SoC } & \text { Museic } & 2.0 \\
\text { (PPG, } & \text { RSP, } & \text { ECG, } \\
\text { EDA) } & & \end{array}$ \\
\hline [21] & $\begin{array}{l}\text { Epileptic seizures } \\
\text { detection }\end{array}$ & $\begin{array}{l}\text { Yes } \\
\text { (Cortex-M4) }\end{array}$ & $4 \mathrm{~s}$ & $\begin{array}{l}\text { RF (2-class, } 54 \text { features) Data } \\
\text { fusion of two moments of time }\end{array}$ & Gmean: $94.5 \%$ & $\begin{array}{l}40.87 \mathrm{~h} \\
(300 \mathrm{mAh})\end{array}$ & e-Glass (2-EEG) \\
\hline [22] & $\begin{array}{l}\mathrm{CW} \text { in rescue mis- } \\
\text { sion }\end{array}$ & No & $60 \mathrm{~s}$ & $\begin{array}{l}\text { RFECV (24 Features), XG- } \\
\text { Boost (2-class), }\end{array}$ & $\begin{array}{l}\text { Acc: } 80.2 \% \text {, } \\
\text { Gmean: } 75.5 \% \text {, }\end{array}$ & - & $\begin{array}{l}\text { Biopac (PPG, RSP, } \\
\text { SKT, ECG) }\end{array}$ \\
\hline
\end{tabular}

CW: Cognitive Workload; RFE: Recursive Feature Elimination; RF: Random Forest; Feat-Imp: Feature Importance Selection; SVM: Support Vector Machine; SSVEP: brain response elicited during a periodic visual stimulation - brain-computer interfaces (BCIs); Acc: Accuracy

*Estimated for a Cortex-M3 micro-controller (active mode)

humans in simulation tasks of rescue missions with drones with our new wearable device, which achieves a battery lifetime of $14.58 \mathrm{~h}$ and an accuracy of $76.63 \%$, comparable with the state-of-the-art proposals and $2.6 \%$ lower than an analogous offline computer-based analysis.

- We simulate a self-aware approach to exploit different energyscalable modes, which yields an increase of $51.6 \%$ of the battery lifetime (up to 22.11 hours). Furthermore, we achieve this lifetime improvement with an insignificant accuracy loss of $1.07 \%$.

The rest of the paper is organised as follows. Section II gives an overview of related work. Section III presents the proposed system. Section IV describes the hardware and software design. In Section V we validate our system on several levels (from hardware, software, to finally application level), while in Section VI we evaluate our energy-aware approach. Finally, in Section VII we draw the main conclusions of this work.

\section{Related Work}

The cognitive workload is defined as the physical or mental requirements associated with a task or combination of tasks performed under specific environmental and operational conditions, also considering the operator's capabilities to respond to those demands [3]. Hence, to consider the operator's individual needs, cognitive workload level has to be monitored based on the mental and physical state. The cognitive workload can be detected via a combination of different physiological signals that describe the physical and mental state of the operator [3]-[5]. The most frequently used signals are electroencephalogram (EEG), respiration (RSP), electrocardiogram (ECG), pulse wave through photoplethysmography (PPG), electrodermal activity (EDA) and skin temperature (SKT) [7]-[9]. Moreover, when considering the multidimensionality of workload physiological response, it has been demonstrated that the highest detection performance is achievable via a multimodal approach, where accuracy values range between $89-99 \%$ and $80-86 \%$ on cross-validation [9], [11] and unseen test sets [8], [12], [13], [22], respectively.

Multimodal monitoring applications require extensive data handling and processing due to the filtering, signal delineation and feature extraction algorithms employed. The number and type of signals and features used, sampling frequency, and segmentation window length are factors that define application memory needs and computational complexity. Table I summarises multimodal monitoring applications that use either commercial wearable sensors or ad-hoc wearable systems proposed in the literature. It includes the main specifications concerning wearable multimodal monitoring applications: sensor platform, algorithms and methods employed, detection performance, and battery lifetime. All these studies still perform data processing offline on a computer, even when using wearable devices for data acquisition [9]-[13]. Indeed, only recently, a few proposals stream physiological signals to other devices for online processing [23].

Despite the recent explosion of wearable devices and systems, no suitable device exists today for multimodal on-device workload monitoring. In fact, no existing device integrates a comprehensive set of signals needed for $\mathrm{CW}$ detection. Further, wearable devices such as the E4 Empatica wristband [24] and Everion band [25], provide continuous acquisition of different physiological data, but they do not process the data locally. Instead, the data is continuously streamed to their cloud infrastructure, consuming a significant amount of energy. Furthermore, other devices that perform online edge-processing are designed for specific applications, such as the Zephyr BioHarness 3 device for cardiac and biomechanical monitoring [26], and the Smartcardia patch for arrhythmia and cardiac monitoring [27].

More recently, to overcome the challenges of multimodal applications, novel ultra-low-power systems on chip (SoC) that include multi-core processing unit [19], [28] and HW accelerators [20], [29], [30] have been proposed in the literature. However, those SoC solutions for wearable devices do not integrate the required set of signals for cognitive workload monitoring (i.e., ECG, RSP, SKT, EDA, and PPG) [20]. In parallel, application-oriented optimization approaches have been proposed to tackle such challenges as well. For instance, the more common optimizations are based on reducing sampling frequency and precision of the acquired signals, decreasing the set of features, using less complex models, etc.

Moreover, smart algorithms have been recently proposed to exploit trade-offs between the required accuracy of the embedded application and the system battery lifetime by applying the system self-awareness concept for edge wearable devices [13]-[16]. It has been shown that a simple classifier, although less energy-hungry, cannot always provide high detection performance. On the other hand, a more complex classifier, while providing high detection performance, reduces energy efficiency significantly. Therefore, previous works have proposed to dynamically employ a reduced number of features whenever the system can provide high confidence information and switching to a high-dimensional feature set only when required.

The design of an online multimodal bio-monitoring system for 


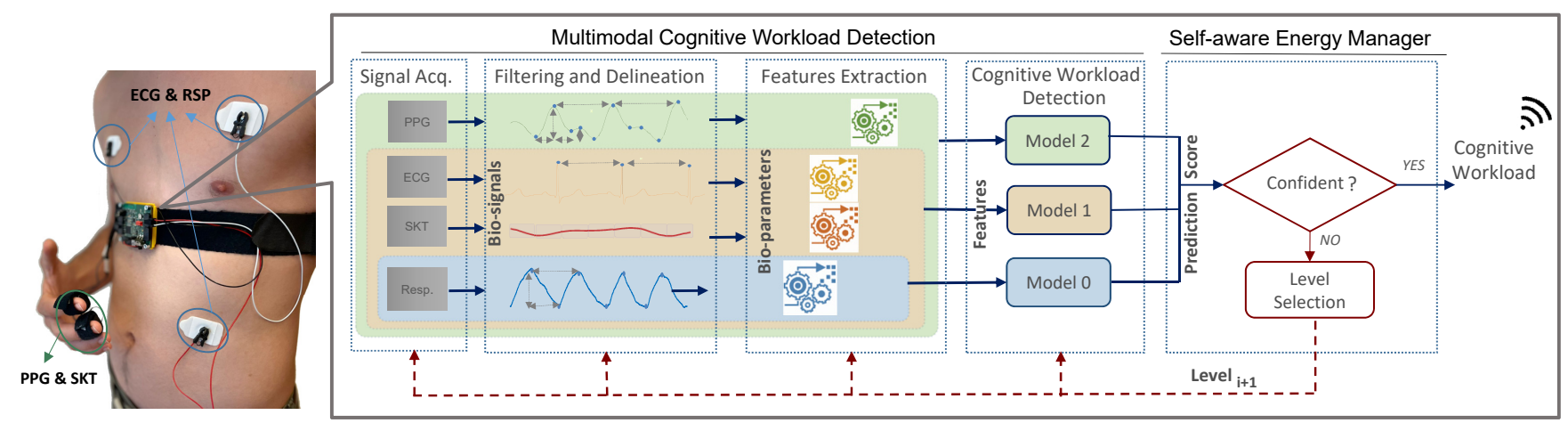

Fig. 1: MBioTracker: Proposed self-aware embedded system processing flow for cognitive workload monitoring.

wearable devices with edge-processing encompasses several requirements and challenges, which are mainly defined by the application and HW platform constraints/resources [13], [21], [31]. In our case, based on the literature review, cognitive workload monitoring requires the acquisition of ECG, PPG, RSP and SKT signals, a segmentation window of 60 seconds and a feature set that efficiently describes the state of the person. Finally, to meet the constraints imposed by the limited battery lifetime and memory size, smart algorithms and a low complexity machine learning model are employed. In this regard, to find a trade-off between classification performance and energy efficiency, the concept of self-aware monitoring is considered.

\section{Self-AWARE AND ENERGY-ScALABLE ONLINE WORKLOAD MONITORING SYSTEM}

The self-aware and energy scalable wearable embedded system, MBioTracker, is designed to enable multimodal online monitoring of cognitive workload. To this aim, we propose a customized system incorporating both off-the-shelf hardware (HW) and novel software (SW) solutions. Our new system includes, on the HW side, a multichannel physiological signals acquisition and a low-power processing platform using state-of-the-art components, which are detailed in Section IV Secondly, on the SW side, a novel multimodal bio-signal processing methodology is utilized, which addresses online filtering, delineation, and feature extraction. Moreover, MBioTracker employs scalable energy embedded machine-learning algorithms and methods for an online person's state monitoring by applying the system selfawareness concept, similar to [13], [14].

An overview of our system design is shown in Fig. 1. The main components are highlighted by dashed lines. First, the sensor block integrates different front-ends for the bio-signals acquisition stage. Second, in the multimodal signal filtering and delineation stages, signals are segmented, filtered and delineated to obtain the bioparameters that better describe the physiological response of the person's state. Those bio-parameters are processed in the feature extraction stage to calculate features that provide information about the physiological response dynamics. Next, in the classification stage, the pre-trained machine learning models provide a prediction score that is analyzed using Self-Aware Energy Manager to select the operation mode for the next detection cycle. Finally, an estimation of the current cognitive workload level of the user is provided and can be sent, e.g., via Bluetooth Low-Energy to a different device.

The main idea behind our Self-Aware energy-management methodology is that the system by default detects the cognitive workload state with a smaller and simpler set of features and machine-learning model. Hence, the model with minimal energy consumption and acceptable detection performance is defined as Level 0 of operation. Then, based on the confidence level of the detection, the system continues in Level 0 or, if necessary, switches to a higher level (i.e. a larger, more complex feature set and model) for the next detection cycle. The detection confidence is estimated with a non-binary score (e.g., likelihood, detection probability, or similarity score), indicating the probability that a sample belongs to a particular class. In other words, the system dynamically switches between different levels of operation, where an operation level is defined by a specific set of sensors, features and classification model.

Furthermore, the multi-level and multimodal architecture design of MBioTracker enables the selective activation/deactivation of sensors and processing algorithms for each signal independently (i.e. the signal acquisition, filtering and delineation, and features extraction stages) depending on the confidence level of the previous classification. Hence, by exploiting these characteristics, the Self-Aware Energy Manager controls the modular execution of different complexity levels and, consequently, different energy consumption levels. Therefore, our system can work in different scalable-energy levels of operation, improving its energy efficiency while ensuring high classification performance.

\section{A. Multimodal Cognitive Workload Detection}

Following the design requirements of an online multimodal biomonitoring method, we first address the multidimensional aspect of cognitive workload [32]. Our wearable system is designed to extract a set of features from different physiological signals that allow unobtrusive and daily measures, i.e. RSP, ECG, PPG, and SKT. Second, the selected segmentation window to analyze those physiological signals is 60 seconds, as in [7], [12], [13], [22].

1) Dataset: In particular, we target the cognitive workload monitoring of an operator flying a drone during a search and rescue mission, as in [22]. For this analysis, we use the database presented in [8], [22], where the experimental protocol and data collection is described in detail. The ethical approval for this study was obtained from the Cantonal Ethics Commissions for Human Research Vaud and Geneva (ethical approval application number PB2017-00295).

The dataset includes 48 records, consisting of RSP, ECG, PPG, SKT, and EDA signals collected from baseline (BL), medium workload and high workload tasks (CW). It was collected from 24 volunteers ( 6 females and 18 males) aged between 21 and 39 years old (Mean and Std: $27.7 \pm 4.8$ ) [22] that participated in two sessions on different days. Each record entails three trials consisting of BL and a randomized sequence of the cognitive tasks. In Trial 1, a resting period of 3 minutes was enforced after each 5-minute task. Then, in Trail 2 and 3, the tasks lasted 3 minutes and were sequentially performed.

From the dataset, we used only baseline (BL) and high cognitive workload $(\mathrm{CW})$ tasks for our binary classification leading to $22 \mathrm{~min}$ per record. Trials 1 and 2 of each record are used as training data and Trail 3 was used as the test set. Training data was used for feature 


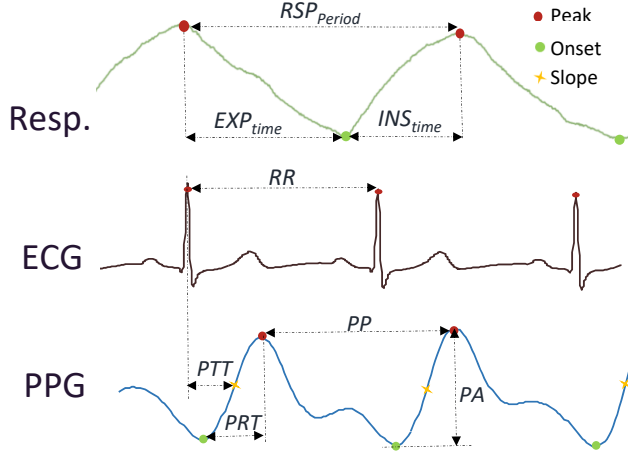

Fig. 2: Delineated points and bio-parameters for each physiological signal.

selection and training of the SVM model with 22 features. Then, it was also used for the selection of optimal thresholds for switching between the self-aware levels. The test set was used to evaluate the final performance on the MBioTracker board and its comparison with the offline (Matlab) performance.

2) Filtering and Delineation: The filtering of the ECG signal happens simultaneously with the acquisition, using the relative energy filtering approach described in [33]. RSP and PPG signals are filtered using bandpass FIR filters of [0.03-0.5] $\mathrm{Hz}$ and [0.1-5] $\mathrm{Hz}$, using orders 10 and 32, correspondingly.

Signal delineation consists of computing the fiducial points (e.g., signal onset, peak, offset, etc.) of the sampled signals, as shown in Fig. 2 For ECG, the relevant points are R peaks, which are further used to calculate $R R$ intervals as a bio-parameter. In the RSP signal, the delineated points are peaks and valleys that correspond to the beginning and end of inspiration and expiration. From the detected points, inspiration and expiration time (INS time and EXP time), as well as respiration rate and period are calculated $\left(R S P_{\text {period }}\right)$. The PPG signal onset, peak, and maximum slope point between the onset and peak are detected, followed by the calculation of the PPG bio-parameters $P P$ (peak to peak time interval), $P R T$ (pulse rise time) and $P A$ (pulse amplitude).

3) Feature selection and Model Training: We extract an initial set of 102 features for workload detection based on [8], [12], [22]. Then, the final set of physiological features used in MBioTracker is selected by Recursive Feature Elimination with Cross-Validation (RFECV), as in [22]. RFECV allows the selection of the most important physiological features that better characterize different levels of cognitive workload. The 22 selected features from PPG, ECG, RSP, and SKT signals are listed in Table II

Both RFECV and the machine learning algorithm are trained and validated on the training set using a shuffled Leave-P\%-Data-Out Cross-Validation with five iterations, considering in each iteration $20 \%$ of data as the validation set. We selected a Support Vector Machine (SVM) due to its suitability for embedded implementations and proven performance compared to other classifiers [5], [22]. An additional strength of the SVM classifier is that it provides a score indicating the likelihood that a sample belongs to a particular class. This score is used as a measure of self-awareness, that is, an estimation of detection confidence.

Finally, using the selected set of 22 features, we train the linear SVM model on the training set and prove its generalization on the test set, obtaining an $80.32 \%$ of accuracy over all subjects (Sensitivity: 74.07\%; Specificity: 86.51\%; F1: 79.37\%; Gmean: 80.05\%)

\section{B. Self-Aware Energy Manager}

The Self-Aware Energy Manager enables the system to adapt itself to specific situations [36], [37] by selecting the most energy-
TABLE II: Selected features for each physiological signal.

\begin{tabular}{ll}
\hline Signal & Features \\
\hline SKT & Average power; Gradient. \\
\hline \multirow{3}{*}{ RSP } & $\begin{array}{l}\text { INStime Mean and Median; EXPtime Median; RSPperiod } \\
\text { Mean; RSPrate Mean, Median and RMS of its first derivative; } \\
\text { BandPowers [0.5-0.75] Hz and [0-0.25] Hz normalised by } \\
\text { the BandPower [0-1] Hz; }\end{array}$ \\
\cline { 2 - 2 } & Respiratory Sinus Arrhythmia (RSA) std [34] \\
& $\begin{array}{l}\text { HF gauss mean frequency of a Gaussian distribution used } \\
\text { to fit the power spectral density estimated in the HF band }\end{array}$ \\
ECG & $\begin{array}{l}\text { [0.15-0.4] Hz of RR values; RR BandPower [0.25-0.3] Hz } \\
\text { normalized by BandPower [0.15-0.4] Hz; }\end{array}$ \\
&
\end{tabular}

PTT normalized HF power [0.15-0.5] Hz

PP Median, BandPower [0.35-0.4] Hz /[0.15-0.4] Hz and Cardiac Sympathetic Index from Lorenz plot [35]; PA RMS and L from Lorenz plot; PRT Median and Pondered

PPG Power $(\mathrm{nLF}+1 / \mathrm{nHF})$ where $\mathrm{nLF}$ and $\mathrm{nHF}$ are the LF band $[0.04-0.15] \mathrm{Hz}$ and HFband $[0.15-0.4] \mathrm{Hz}$ power normalised by the BandPower [0.04-0.4] Hz

efficient operating mode that meets a given confidence threshold on detection. To illustrate this approach in the context of workload monitoring, we propose a case study with three levels of scaled energy embedded machine learning algorithms based on SVMs. However, our approach can be generalized to multiple levels (L) of machinelearning models. The levels can be designed with different trade-offs between energy consumption and the probability of obtaining high detection performance in different situations, as presented in detail in Section VI In particular, the three levels defined for MBioTracker, presented in Fig. 3 are the following:

Level 0 uses features extracted from a single physiological signal, the RSP, whose 9 features are the most important and whose pretrained model has the highest probability of being used (as reported in details in Section VI. At the same time, the use of a single sensor drastically reduces the energy consumption of the system.

Level 1 uses all features except the 8 extracted from PPG. This choice is motivated by the fact that both PPG acquisition and processing are the most energy-consuming (as discussed in Section VI] in this case study.

Finally, Level 2 uses the complete set of selected features (see Table [II and comprehensively detects cognitive workload, hence providing more accuracy in terms of classification accuracy but also having the highest energy consumption.

To switch between the three different levels, we use the likelihood score provided by the SVM. As shown in Fig. 3 when the absolute value of this score is lower than a certain threshold (i.e., close to the separation hyperplane), the classification is considered not confident enough. Consequently, the Self-Aware Manager will select a higher complexity level for the next detection window of analysis. As each level contains all features of lower levels, the Self-Aware Manager will evaluate if, at any point, a simpler model provides sufficient confidence and, if so, will reduce the complexity level in the next detection window.

Our self-aware design differs from previous studies [13], [14]. It selects the level of operation for the next segmentation window, as opposed to switching to more complex models in the same segmentation window of analysis until the system is confident enough about its current detection. Although the solution in [13], [14] has the advantage of assuring the best detection performance at any time, it needs to acquire and store all physiological signals in every window as some may be needed for eventual further processing, reducing energy efficiency. In contrast, we extend the concept of system selfawareness and energy scalable machine-learning system down to the 


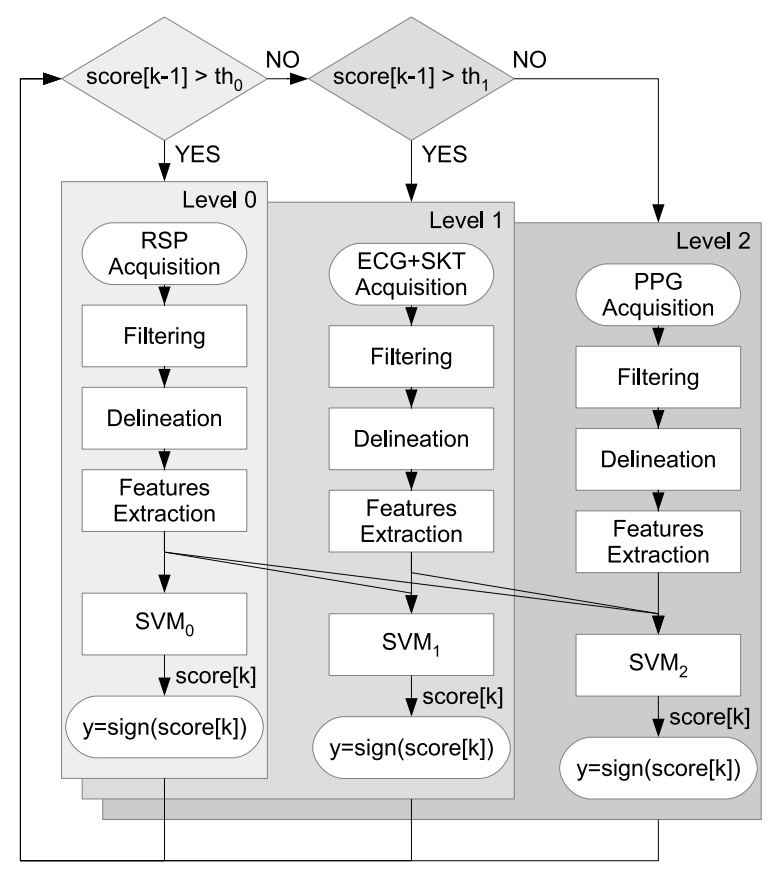

Fig. 3: Self-aware and energy-scalable approach for online cognitive workload monitoring.

acquisition level, proposing a more energy-efficient solution than that demonstrated in the literature. On the other hand, the confidence of our classification is evaluated with one segmentation window of delay, that is, based on the score provided by the previous classification window. In a worst-case scenario, this approach can potentially lead to up to two windows (two minutes) of non-optimal prediction due to usage of the simpler and less confident model while the system increases complexity from Level 0 to 2 . Therefore, our solution may pay the price of improved energy efficiency with a possible drop in detection performance when a sudden change in the cognitive state requires a very complex detection.

\section{Self-aware Operation Levels Configuration and Online Classification}

Each level of operation uses a machine-learning model and a threshold of the prediction confidence defined in the training phase of our self-aware classification procedure. Although our machinelearning algorithm used to detect high levels of cognitive workload is based on a linear SVM (see Section III-A), the presented procedure is not restricted to this particular classifier. In fact, any binary classification technique providing a non-binary score (e.g. detection probability) can be considered as well.

In our case, as we have three levels of scalability, we need to define two thresholds, namely, $t h_{1}$ and $t h_{2}$, which are cross-validated with the training data, see Section III-A.1. The training phase of our self-aware classification procedure is shown in Algorithm 1 and includes two main steps. The first step consists of training the classifiers of each level $(l)$, with the selected training set $\left(\mathbf{X}_{t r}\right)$ and the corresponding ground-truth labels $\left(\mathbf{y}_{t r}\right)$ (lines 2-4). Then, in the second step, the required thresholds are defined (L-1) to switch between two consequent levels (lines $5-23$.

The algorithm iterates over the possible threshold candidates (th) in a range from 0 to th $\mathrm{Max}_{\mathrm{Max}}$ with an increment of 0.05 , where $\mathrm{th}_{\mathrm{Max}}$ is the maximum absolute confidence score given by the classifier (line 6). Hence, for each threshold value, we determine the accuracy using $N_{\text {folds }}$ (5 in our case) cross-validation (lines $8-17$ ). In each fold of the cross-validation procedure, the accuracy for each threshold
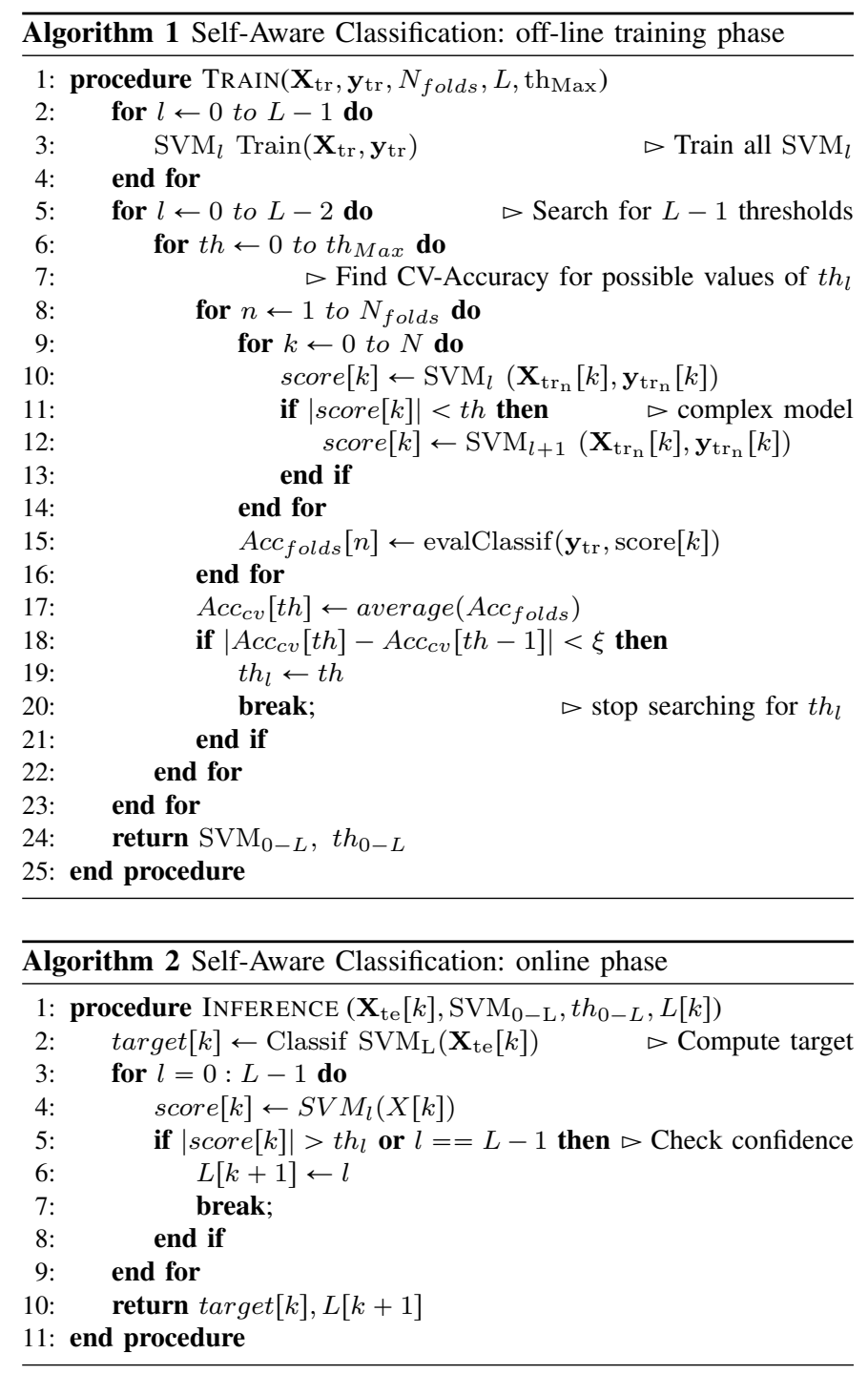

value $\left(A c c_{c v}[t h]\right)$ is determined by iterating through all samples and calculating their scores, always with the lower-level model first. Only in the case of a score being smaller than the currently considered threshold do we switch to a higher level classification model (lines 10-13). Therefore, lower thresholds result in a lower probability of switching to a higher level, which significantly improves the final MBioTracker energy efficiency.

Optimal classification accuracy is defined as the accuracy level at which increasing the threshold does not significantly improve (specifically, more than $\xi$ ) the accuracy values, i.e., when we reach a plateau of accuracy. Therefore, when we reach a plateau, we save the threshold as the optimal threshold between model levels $l$ and $l+1$ and terminate the threshold search (lines 18-20. Once the energy-scaled models of each level are trained and the thresholds indicating the confidence bounds are defined, the full self-aware machine-learning model is prepared for inference. The online phase of our classification procedure is shown in Algorithm 2 First, the algorithm processes the $k^{t h}$ observation $X[k]$ to compute the current inference target $[k]$ (line 2) using the SVM model that corresponds to the selected level $L[k]$ in the previous inference cycle. Next, the level for the next detection cycle is selected $L[k+1]$ (lines $3-9$ ). The algorithm always starts by checking if the confidence of level 0 is higher than threshold 0 (line 5). If this is not the case, the inference is not confident enough and a higher level must be selected. The 

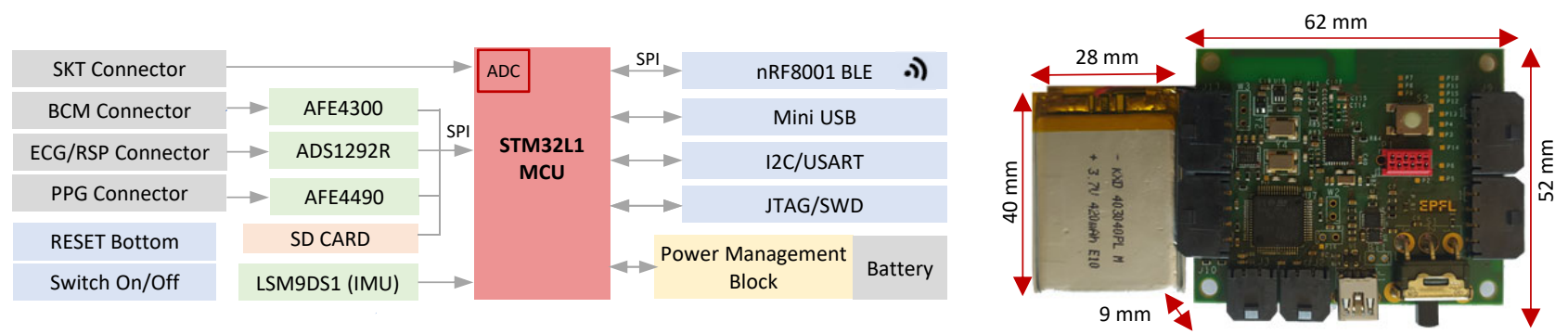

Fig. 4: Block diagram and prototype of the proposed MBioTracker wearable system.

algorithm repeats the process from line 4 until a sufficiently high confidence level is found or the highest level is selected. Finally, the algorithm returns the values of the current segment inference and the selected level for the next inference cycle (line 10).

\section{SYSTEM DESIGN AND IMPLEMENTATION}

We have designed and implemented the new MBioTracker wearable system using a validated set of commercial off-the-shelf components intended for RSP, PPG, ECG, Impedance Cardiography (ICG), EDA, and SKT signal acquisition. The prototype of the proposed wearable system is shown in Fig. 4 The principal components are highlighted, namely, the Micro-Controller Unit (MCU) for computing and power management, the biosignals sensors, an Inertial Measurement Unit (IMU), a Bluetooth Low-Energy (BLE) module for communication, and an SD Card for onboard data storage. The IMU that includes a 3-axis accelerometer, gyroscope, and magnetometer can be used for signal artifacts removal. Furthermore, the platform includes different communication technologies (e.g., BLE, I2C, USART), allowing interconnections with external devices, such as a PC, tablets, smartphones, or even smartwatches.

For online data processing, the board utilizes an Ultra-Low-Power 32-bit MCU Arm Cortex-M3 (STM32L151xD) [38] core with a flexible CPU clock from $32 \mathrm{kHz}$ up to $32 \mathrm{MHz}$. The board also includes the nRF8001 BLE Radio Frequency transceiver [39], which can be used to communicate with a tablet, smartphone, or smartwatch.

Table III presents the selected main components of our system and its characteristics. It includes off-the-shelf components available in the market for single unit, and it can be considered as an upper bound of the final possible cost when the system is industrialized for large production volumes. The following sections describe the bio-sensing modules for acquiring the physiological signals and the hardware power-saving modes used during run-time.

\section{A. Bio-Sensing Modules}

The bio-sensing modules include voltage and optical sensors connected to the MCU through a Serial Peripheral Interface (SPI). Table III presents the selected components and configuration for each signal sensor of our monitoring system. First, it includes a 16-bit Analog Front-End (AFE4300) [40] for Body Composition Measurement (BCM) used for measuring body impedance, as well as ICG, EDA, and RSP. Second, it incorporates an analog temperature sensor (LMT70) [41] that is connected to the 12-bit ADC of the MCU for SKT measurements. An additional Analog Front-End (AFE4490) [42] is mounted for Photoplethysmography (PPG) and pulse oximetry. Finally, 24-bit Analog Front-End (ADS1292R) [43] is used for both RSP and ECG signals measurements, as it includes two configurable channels.
TABLE III: MBioTracker components and characteristics for current prototype development.

\begin{tabular}{|c|c|c|c|c|}
\hline \multicolumn{2}{|c|}{ Component } & \multicolumn{3}{|c|}{ Characteristics } \\
\hline \multicolumn{2}{|c|}{ Bio-sensors } & fs & ADC Range & Precision \\
\hline ECG & ADS1292 [43] & $8 \mathrm{kHz}$ & $\pm 2.42 \mathrm{~V}$ & 24 bits \\
\hline RSP & ADS1292 [43] & $8 \mathrm{kHz}$ & $2-10 \mathrm{k} \Omega$ & 24 bits \\
\hline PPG & AFE4490 [42] & $5 \mathrm{kHz}$ & $\pm 1.2 \mathrm{~V}$ & 22 bits \\
\hline SKT & LMT70 [41] & $1 \mathrm{MHz}$ & $20-42^{\circ}$ & 12 bits \\
\hline $\mathrm{BCM}$ & AFE4300 [40] & $860 \mathrm{~Hz}$ & $0-2.5 \mathrm{k} \Omega$ & 16 bits \\
\hline IMU & LSM9DS1 & $1 \mathrm{kHz}$ & $1.9-3.6 \mathrm{~V}$ & 16 bits \\
\hline $\mathrm{MCU}$ & STM32L151xD & \multicolumn{3}{|c|}{ 32-bit Arm Cortex-M3 at $32 \mathrm{MHz}$} \\
\hline BLE & nRF8001 & \multicolumn{3}{|c|}{ V4.0 } \\
\hline
\end{tabular}

\section{B. Power Management System}

To ensure the low-power usage of the proposed system, we included a power management approach that involves control of both the MCU and its peripherals. The power modes of both the MCU and the sensors are handled via the three defined levels of operation of our system (i.e., Level 0, Level 1, and Level 2) controlled by our Self-Aware Energy Manager (cf. Sec. III-B). To this aim, and to achieve the best compromise between low energy consumption and short startup time, we utilize three main operational modes on the MCU, namely, Run mode, Sleep mode, and Stop mode. Run mode is the normal operating mode, where all the required resources of the MCU are active, with a current consumption of $10.5 \mathrm{~mA}$. In Sleep mode, the CPU is stopped, while all resources of the MCU continue to operate and can wake up the CPU in $0.4 \mu \mathrm{s}$ in the event of an interrupt. Sleep mode power consumption is about $2 \mathrm{~mA}$ with all peripherals off. Finally, Stop mode achieves the lowest power consumption, less than $1.35 \mu \mathrm{A}$, while retaining the RAM and register contents and real-time clock. The MCU can be woken up in $8 \mu$ s by interrupts or events on specific external lines.

The Run mode is switched to the power saving modes (i.e., Sleep and Stop modes) when the MCU is not processing data. The selection of the power saving mode for each operation level depends on the sampling frequency of the active sensors, the processing time of that level and the time required to reactive the elements of the MCU for specified power mode. In particular, the Sleep mode is used for Level 1 and Level 2 since only the CPU is stopped, while all peripherals continue to operate and can wake up the CPU when an interrupt occurs. Stop mode is used for Level 0 since only the RSP signal is sampled and the $8 \mu s$ wake-up time from Stop mode is short enough to service an interrupt from the RSP front-end.

Peripheral power management (e.g., sensors, SD card, BLE module) is handled from the MCU. The power modes of the different peripherals, with the corresponding current consumption and wakeup time, are considered to switch them on and off based on the operation level of the system. In this regard, the MCU's features, such as timers and customized interrupt lines, simplify the design, allowing the implementation of event-driven applications. 
TABLE IV: Distributed processing using interrupts and timers.

\begin{tabular}{lcccc}
\hline Processing step & SKT & ECG & RSP & PPG \\
\hline Acquisition & ADC1 $(5 \mathrm{~Hz})$ & EXTI2 $(125 \mathrm{~Hz})$ & EXTI2 (5Hz) & EXTI3 (64Hz) \\
Filtering & - & EXTI2 (125Hz) & TIM7 (3s) & TIM7 (3s) \\
Delineation & TIM10 (1s) & TIM6 (1.75s) & TIM7 (3s) & TIM7 (3s) \\
Feature extraction and classification & TIM5 (60s) & TIM5 (60s) & TIM5 (60s) & TIM5 (60s) \\
\hline
\end{tabular}

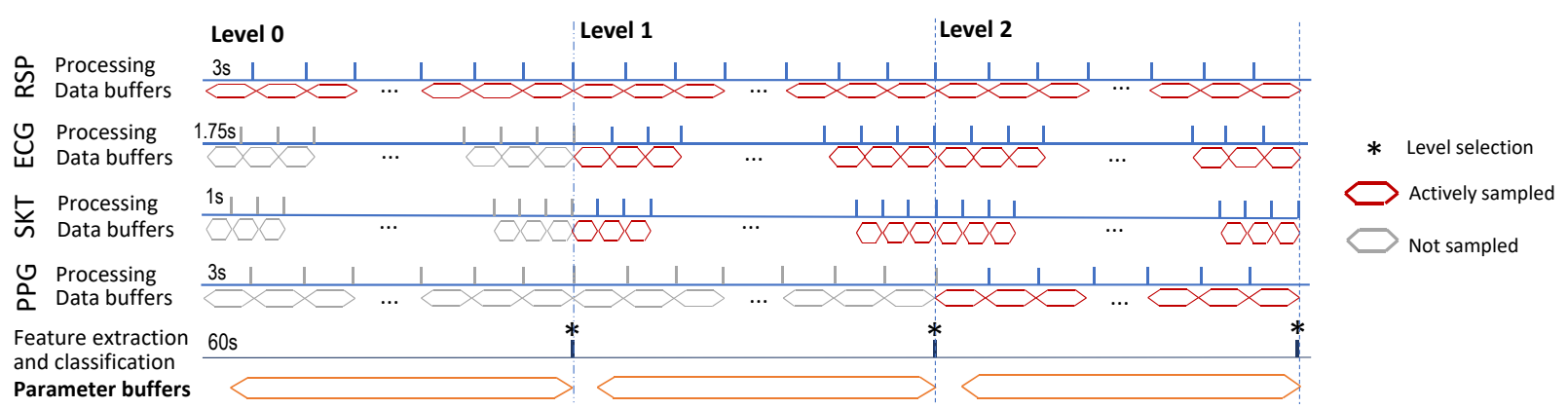

Fig. 5: Timing diagram of sampling, delineation and feature calculation.

\section{Real-Time Software Design}

The proposed SW architecture for workload detection is shown in Fig. 1 in Sec. III The system needs to process four signals (i.e., PPG, ECG, RSP, and SKT). To this end, we propose a five-stage SW structure, which includes signal acquisition, processing (i.e., filtering and delineation), feature extraction and classification. Each stage is activated by interrupt requests triggered by the front-ends for the acquisition stage and timers for other stages. The acquisition stage has the highest priority, while the feature extraction and classification stages have a low-priority interrupt associated with a 60-second timer.

Moreover, to maintain application flexibility and allow future parallelization, the processing of each signal is managed independently of each other. The objective is to create an independent and modular signal processing implementation that can be used in other contexts and applications. Thus, the different stages of the software are distributed into separate routines, at least one for each stage and signal. The overview of all processing steps for each signal, the timers/interrupts that trigger them, and corresponding activation frequencies are shown in Table IV] and discussed below.

1) Signal Acquisition: The first stage of the system architecture is signal acquisition representing the software interface between the MCU and the front-end devices. In this stage, the data from every signal is acquired, converted to a standard datatype, and stored in raw data buffers, called acquisition buffers.

Data conversion standardizes the sample datatype for the following stages since each front-end has its own data type and precision. This is applied to allow the reuse of functions that are common among signals. Thus, we convert the samples into a signed 16-bit integer data type, thus reducing memory usage and computational cost compared to higher precision datatypes such as floating-point.

The acquisition buffers consist of a set of static-length circular buffers, whose objective is to prevent the system from overwriting the data before being processed. Hence, the established length of each signal's buffer is determined by the sampling frequency and the processing window required by the processing stage. The three frontends are working asynchronously. Each signal acquisition is handled by an interrupt service routine called by the respective front-end's interrupt request every time a sample is available. All the signals are assigned the highest priority of the SW system. Thus, the SW system acquires an available signal sample regardless of the task currently processed, avoiding data loss during acquisition.
2) Multimodal Signal Filtering and Delineation: Filtering removes noise and artifacts from the physiological signals by applying a digital filter. Signal delineation consists of computing the fiducial points (e.g., signal onset, peak, offset, etc.) of the sampled signals. As shown in Table IV the ECG signal filtering happens simultaneously with the acquisition, while RSP and PPG filtering is done every 3 seconds, just before the delineation phase occurs. The implementation was done using the CMSIS-DSP library in order to lower the computational cost and time. Filtered data is stored in circular buffers so that it can be used later by the delineation algorithms.

In the final delineation step of this phase, fiducial points relevant for further bio-parameters calculation are detected, as shown in Fig. 2 and explained in Sec. III-A.2 Delineated points and bio-parameters are stored in circular buffers, called bio-parameters buffers, containing different data structures depending on the physiological signal. The length of these bio-parameters buffers depends on the maximum physiological occurrence rate of the delineated points.

3) Features Extraction and Classification: Finally, once the feature extraction timer is triggered (every 60s), physiological features are calculated using data from the bio-parameter buffers collected in the last 60s. Then, features are saved in a structure containing all features and stored in a circular buffer. As presented in Sec. IIIA.3 and Table II the features set includes simple statistical features (e.g., mean, median, RMS) and more complex ones in the frequency domain (e.g., normalized power in specific frequency bands), which are computed using functions from the CMSIS-DPS library as well. After the features are calculated, normalization is applied using pre-calculated values from the training set. Finally, classification is performed using the pre-trained SVM, thus leading to a workload classification result every 60 seconds.

Finally, we decide for the self-aware level of the next one minute, and hence, the physiological signals that need to be sampled and the algorithms and interrupts that should be active. It is done based on the confidence values of the current level model and the simpler level models (if any) with data of the current classification window.

4) System Timing and Synchronizing Description: We propose a system structure by stages (acquisition, filtering and delineation and, finally, features extraction and classification), where each stage is triggered by a corresponding interrupt or timer defined by the stage type and the physiological signal. Furthermore, outputs of each signal's stage are stored in circular buffers until the next stage uses them as an input, as shown in Fig. 5 In particular, the data buffers store the signals until it is processed (filtering and delineation, where 
parameters are calculated) and then, stored in each signal's parameters buffer.

Furthermore, using our proposed modular design, use of interrupts, as well as including circular buffers with pointers that are keeping track of data being processed, we implemented a scheme that enables us to synchronize data between different signal modules. This scheme is especially important for Respiratory Sinus Arrhythmia (RSA) and Pulse Transit Time (PTT) features. In particular, when we calculate RSA features that require synchronized RR values (from ECG) with the RSP signal, we keep track of the time stamp of each RR value as well as RSP data points and then interpolate the RR signal to the same time stamps as RSP data.

Due to the long prediction period of our application (i.e., $60 \mathrm{~s}$ ) and relatively long windows for the intermediate stage of filtering and delineation (i.e., 1.75-3 s), our system can easily cope with processing time requirements. Total processing time and time stages are in the order of milliseconds, as reported in Sec. V-C Table VIII which leads to a very low duty cycle of our system. Thus, our system structure guarantees no data loss and delays in the real-time application. Also, our approach guarantees the synchronization of different physiological signals.

\section{System EVALUATION}

We have evaluated and assessed our new system at three levels of abstraction, namely, 1) on-board sensors and signal acquisition, 2) signal processing and feature extraction algorithms, and 3) application level. Moreover, we present the computational complexity and memory usage of our system for different physiological signals.

\section{A. Multi-Channel Acquisition Platform: Hardware Validation}

We compare the signals acquired through our system with those obtained from a validated acquisition system from Biopac [44]. We consider the ability to delineate the signals' fiducial points as a metric for evaluating the signals' quality [45]. To this aim, we synchronously acquired 23 minutes of signals with both our system and the Biopac. The delineated points from both signals were compared. Every delineated point from our board found within a resolution window around the reference (Biopac signal) delineated point is considered true positive (TP) and otherwise as false positive (FP). If no points are found within the resolution window, we count a false negative (FN). The resolution windows are $\pm 20 \mathrm{~ms}$ for ECG and PPG, and $\pm 400 \mathrm{~ms}$ (2 samples) for RSP.

The evaluation metrics comprise sensitivity calculated as $\mathrm{TP} /(\mathrm{TP}+\mathrm{FN})$, predictivity as $\mathrm{TP} /(\mathrm{TP}+\mathrm{FP})$ and mean error as distance in seconds between matched peaks from both signals. Table $\mathrm{V}$ reports the results obtained over the 23 segmented windows of 60 seconds each. As can be seen, the ECG metrics highlight a high-quality detection of the R-peaks having only $12 \mathrm{~ms}$ of difference from Biopac ones. The quality of PPG suffers a slight degradation, but it only affects the final classification by up to $2 \%$. This estimation was obtained by perturbing the inputs of the classifier with errors of such amplitude, and the classifier showed a minimal variation of the result.

Finally, the RSP points' sensitivity and mean error have the lowest sensitivity $(78 \%)$ and the highest error of $0.5 \mathrm{~s}$ in relation to the reference signal. These errors result from the fact that our system measures RSP from the thoracic impedance variations. In contrast, the reference Biopac system utilizes a strap stretched around the chest to capture both expansion and contraction of the rib cage. Furthermore, differing sampling frequencies between the proposed board (RSP at 5 $\mathrm{Hz}, \mathrm{SKT}$ at $5 \mathrm{~Hz}, \mathrm{PPG}$ at $64 \mathrm{~Hz}$, ECG at $125 \mathrm{~Hz}$ ) and Biopac system $(2 \mathrm{kHz})$ may also result in variations between measured MBioTracker and Biopac signals.
TABLE V: Signal quality evaluation by the ability to delineate the fiducial points delineated from the signal acquired with MBioTracker against signals acquired with Biopac system.

\begin{tabular}{lccc}
\hline Fiducial points & Sensitivity (\%) & Predictivity (\%) & Mean error (s) \\
\hline ECG R-peaks & $96.4 \pm 8.70$ & $97.6 \pm 3.60$ & $0.012 \pm 0.004$ \\
PPG peaks & $84.8 \pm 12.9$ & $85.9 \pm 11.2$ & $0.041 \pm 0.037$ \\
PPG onsets & $85.3 \pm 12.9$ & $86.5 \pm 11.3$ & $0.040 \pm 0.037$ \\
RSP peaks & $77.1 \pm 22.2$ & $93.1 \pm 15.1$ & $0.525 \pm 0.334$ \\
RSP onsets & $78.7 \pm 19.4$ & $94.0 \pm 11.7$ & $0.518 \pm 0.333$ \\
\hline
\end{tabular}

TABLE VI: Quality of fiducial point delineation with online on the board and offline Matlab implementation for each physiological signal.

\begin{tabular}{lccc}
\hline Delineated points & Sensitivity $(\boldsymbol{\%})$ & Predictivity $(\boldsymbol{\%})$ & Mean error (s) \\
\hline ECG R peaks & 95.9 & 98.3 & 0.008 \\
PPG onsets & 89.7 & 100 & 0.009 \\
PPG peaks & 89.7 & 100 & 0.006 \\
RSP valleys & 84.9 & 93.2 & 0.104 \\
RSP peaks & 84.8 & 91.8 & 0.052 \\
\hline
\end{tabular}

\section{B. Multi-Channel Acquisition Platform: Software Validation}

To validate the implemented algorithms, we emulate an online cognitive workload detection from the test set of the dataset prerecorded using the Biopac system introduced in Section III-A.1 For emulating real-time signal acquisition, interrupt routines trigger the data sampling from an SD Card instead of sampling from sensors. For this validation, the signals were down-sampled and scaled to meet the characteristics of MBioTracker's acquisition layer.

First, we validated the filtering and the delineation of the fiducial points extracted from RSP, ECG, and PPG signals by comparing peaks detected on board and those detected offline, via a Matlab implementation. The median values for sensitivity, predictivity and mean error of all data and delineated points are shown in Table VI

In the second stage, we validated the features calculated every $60 \mathrm{~s}$ using the points and bio-parameters delineated from the physiological signals. For this analysis, we report the measurements of the relative error and Spearman's correlation coefficient between feature values computed in MBioTracker and their expected reference computed offline. To do this, features values were concatenated for all 60swindows of the test data.

Our results are shown in Fig. 6 as a boxplot representing the distribution over all features (22 values). Almost all features had satisfyingly high similarity and correlation values with a median of Spearman correlation being 0.79 and relative distance of feature values $5.1 \%$. However, more challenging features do not have a significant correlation since they require synchronization between multiple signals (normalized band power of PTT and STD of RSA) or complex calculation (such as PP power band). Further, the differences between the online and offline system are enhanced when multiple effects are combined. These effects include much lower sampling
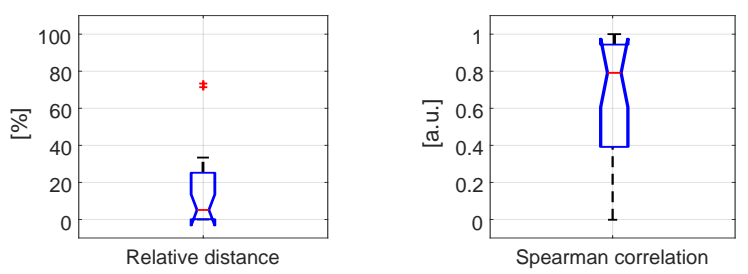

Fig. 6: Feature extraction evaluation over the 22 features. Relative error and Spearman's correlation between feature values computed in MBioTracker and their expected reference computed offline. 
TABLE VII: Memory usage (bytes) for the buffers at the different processing stages.

\begin{tabular}{ccccc}
\hline Signal Acquisition & $\begin{array}{c}\text { Delineation } \\
\text { (Nr. Parameters) }\end{array}$ & $\begin{array}{c}\text { Features Extraction } \\
\text { (Nr. Features) }\end{array}$ & Total \\
\hline ECG & 1000 & $600(1)$ & $16(2)$ & 1616 \\
PPG & 1200 & $2400(3)$ & $64(8)$ & 3664 \\
RSP & 1000 & $1440(4)$ & $80(10)$ & 2520 \\
SKT & 2 & $4(2)$ & $16(2)$ & 22 \\
\hline Total & 3202 & 4444 & 176 & 7822 \\
\hline
\end{tabular}

TABLE VIII: Computation time (ms) of a 60 -second signal processing window.

\begin{tabular}{lccccc}
\hline Signal & \multicolumn{2}{c}{ Processing } & \multicolumn{2}{c}{ Features Extraction } & Total \\
& Filter & Delin. & Time domain & Freq. domain & \\
\hline SKT & 10.4 & 0.078 & 0.046 & - & 10.52 \\
RSP & 8.16 & 5.28 & 1.50 & 6.18 & 21.13 \\
ECG & 118.4 & 12 & - & 20.65 & 151.1 \\
PPG & 139.2 & 95.2 & 14.45 & 29.37 & 278.2 \\
\hline Total & \multicolumn{2}{c}{388.7} & \multicolumn{2}{c}{72.20} & 461 \\
\hline
\end{tabular}

frequency on the MBioTracker, the utilization of 16-bit fixed-point values instead of floating-point, and the online processing in short discrete windows with possible slight desynchronization between online and offline approaches.

Finally, we validate MBioTracker at the application level. We validate the complete system by comparing cognitive workload classification computed online by our proposed system versus the offline PC (Matlab) based implementation of all algorithms. The final accuracy achieved on the test set using our system MBioTracker is $76.63 \%$ (Sensitivity: 69.63\%; Specificity: $84.13 \%$; F1: $75.50 \%$; Gmean: $76.54 \%$ ), which is only $2.6 \%$ lower than the PC based analysis of $79.25 \%$ (Sensitivity: $77.04 \%$; Specificity: $81.75 \%$; F1: 79.39\%; Gmean: $79.36 \%$ ). Performance values were calculated from concatenated predictions every 60 s for all subjects while employing pre-trained personalized SVM models on the board.

\section{Computational Complexity, Memory Usage and Current Consumption}

The memory usage of the processed signals is reported for different processing stages in Table VII The computation complexity of the implemented algorithms is satisfyingly low. We evaluated it by measuring the time required to process the physiological signals, i.e., filtering and delineation, as well as the time required to extract both time and frequency domain features per signal. To this aim, we used the Simplicity Studio software energy profiler on a Cortex-M3 based EFM32LG-STK3600 board. This setup mounted an EFM32LG TM Leopard Gecko 32-bit microcontroller unit (MCU) with 3V supply, comparable with our system. Table VIII reports the time required by the MCU to process the 60s segmentation window of acquired data.

The total computation time per segmentation window for signal processing and feature extraction is $461 \mathrm{~ms}$, only $0.77 \%$ of the duty cycle, guaranteeing no delays and data loss in the real-time application. Moreover, we can see that PPG feature extraction requires the majority of computational time, followed by features extracted from ECG, $60.24 \%$, and $32.77 \%$ of the time, respectively. This can be translated to an energy consumption of only $1.94 \mu \mathrm{Ah}$ and 1.05 $\mu \mathrm{Ah}$ when the MCU is active for PPG and ECG, respectively.

The average supply current of the different components operating under different modes was measured. While the MCU is running and all the sensors are powered down, our system has an average current consumption of $25.1 \mathrm{~mA}$. This current consumption is reduced to 17.4 and $13.8 \mathrm{~mA}$ while in sleep and stop modes, respectively. The current consumption increases by activating the RSP/ECG acquisition
TABLE IX: CV accuracy vs energy consumption of reduced models.

\begin{tabular}{|c|c|c|c|c|c|c|}
\hline \multicolumn{4}{|c|}{ Active Signals } & Features & CV Accuracy & Energy* $^{*}(\mu \mathbf{A} \mathbf{h})$ \\
\hline RSP & PPG & ECG & SKT & 22 & $88.33 \%$ & 159.88 \\
\hline RSP & PPG & ECG & - & 20 & $88.18 \%$ & 159.81 \\
\hline RSP & PPG & - & SKT & 19 & $87.44 \%$ & 158.83 \\
\hline RSP & - & ECG & SKT & 14 & $86.92 \%$ & 17.94 \\
\hline- & PPG & ECG & SKT & 13 & 83.51 & 157.73 \\
\hline RSP & - & - & - & 9 & $84.02 \%$ & 16.81 \\
\hline - & PPG & - & - & 8 & $72.45 \%$ & 141.94 \\
\hline - & - & ECG & - & 3 & $77.30 \%$ & 17.72 \\
\hline - & - & - & SKT & 2 & $71.65 \%$ & 0.07 \\
\hline
\end{tabular}

(ADS1292R) and the PPG acquisition (AFE4490+ LEDs) approximately 1 and $8.4 \mathrm{~mA}$, respectively. These measurements align with the values reported in the different components' datasheets [42], [43].

\section{Self-Aware Power Manager Evaluation}

The multi-level approach for energy scalability and self-awareness of our system, presented in Section III] enables the control of the trade-off between detection performance and energy consumption according to the constraints of our online cognitive workload detection application. To evaluate this trade-off, we first analyzed the workload detection sensitivity of our system for different configurations defined by the levels of operations (Levels 0 to 2, as described in Section IIIB). Then, we measured the energy consumption of the system at each level and estimated the battery lifetime.

\section{A. Energy-Scalable Levels Setup}

In our experiments, we implemented the three energy-scalable levels described in Section III-B namely, a single-signal level (Level 0 ), an intermediate level (Level 1), and the complete model using all signals and the 22 extracted features (Level 2).

To choose which features and signals to be used per level, we tested a single module (only using RSP, ECG, PPG, or SKT features), as well as the full model with 22 features, and then analyzed the effects of removing one signal module from the full model. This is shown in Table IX, where we report the classification accuracy for cross-validation on the training set (see Section III-A.1. Further, in this table, we report the energy consumption of the possible models considering both the energy for signal acquisition and data processing. The data processing energy values were calculated using the EFM32LG TM Leopard Gecko 32-bit MCU. The energy for the signal acquisition was estimated by measuring the MBioTracker current while switching on and off the main components (MCU, RSP/ECG sensor, PPG sensor (including the LEDs driving current), and BLE module), enabling the estimation of the supply current required for each module.

We can see that removing one single signal leads to a similar drop in accuracy of approximately $1.5 \%$, except when removing RSP, which leads to a performance drop of $4.82 \%$. On the other hand, the energy consumption when removing the PPG is considerably reduced, by $88.78 \%$, whereas it is reduced by less than $1.35 \%$ for the other signals. These results motivate the fact that we can drastically increase the battery lifetime by removing PPG without compromising the classification accuracy. Hence, we selected for Level 1 in our MBioTracker design, the model consisting of all signals but PPG.

In contrast, by analyzing the single-signal models in Table IX we observed that the model using the RSP signal has the highest accuracy (more than 6.7\% higher than the other single-signal models). These results suggest that the RSP signal contributes the most to high classification accuracy, which can be explained by the fact that it contains almost half of the features. In addition, it has significantly 
TABLE X: Average energy consumption of the MBioTracker's components while operating the system at different levels.

\begin{tabular}{llccc}
\hline Module & & L0 & L1 & L2 \\
\hline MCU + & Active Mode $(\mu \mathrm{Ah})$ & 0.147 & 1.295 & 3.246 \\
Active comp. & Stop Mode $(\mu \mathrm{Ah})$ & 229.9 & - & - \\
& Sleep Mode $(\mu \mathrm{Ah})$ & - & 289.1 & 287.7 \\
\multirow{2}{*}{ SSP/ECG } & ADS1292R active $(\mu \mathrm{Ah})$ & 16.67 & 16.67 & 16.67 \\
PPG & AFE4490 active $(\mu \mathrm{Ah})+$ & - & - & 140.0 \\
& AFE4490 standby $(\mu \mathrm{Ah})$ & 6.667 & 6.667 & - \\
\hline \multirow{5}{*}{ MBioTracker } & Duty cycle $(\%)$ & 0.04 & 0.30 & 0.77 \\
& Wake-up Period(ms) & 2.56 & 3.07 & 4.61 \\
& Average Current $(\mathrm{mA})$ & 16.29 & 20.12 & 28.80 \\
& Average Power (mW) & 46.46 & 57.32 & 97.42 \\
& Energy ( $\mu$ Ah) & 253.4 & 313.7 & 447.7 \\
& Battery life -420mAh- (h) & 25.78 & 20.87 & 14.58 \\
& Detection Accuracy* $(\%)$ & 62.23 & 68.09 & 80.32 \\
\hline
\end{tabular}

+ including the LED driving current, ${ }^{*}$ from the test set data

lower energy consumption with respect to the full Level 2 model. Thus, we selected the single model that includes RSP features for Level 0 in MBioTracker.

\section{B. Operation Levels Evaluation}

To evaluate our energy-aware strategy, we first analyze the average energy consumption of the different components of our new multimodal wearable device for the operational levels reported in Table $\mathrm{X}$ We consider the computational time while operating in Level 0, Level 1 , and Level 2, leading to duty cycles of $0.04 \%, 0.30 \%, 0.77 \%$, respectively. The energy consumption of the $\mathrm{MCU}$ in processing time increases with the level of operation, but its contributions are significantly lower compared to idle time. The final active time of the MCU also includes a switching period since the MCU needs time to wake up from or to enter into the low-power mode. The energy consumption of the system in idle time is similar for Levels 2 and 1, where the MCU is in sleep mode. On the other hand, we observe that Level 0 benefits from the MCU stop mode. The sleep mode seems to be more appropriate for Levels 1 and 2 due to the relatively high sampling frequency of the active sensors and the required wake-up time of the MCU.

Moreover, we report in Table $\mathrm{X}$ the total energy consumption, the average current, and average power per level. The battery lifetime is estimated by its capacity (a battery of $420 \mathrm{mAh}$ was used as an example) and the total supplied current. Our results show that Levels 0 and 1 are more efficient in terms of energy consumption, which leads to a clear increase in the battery lifetime in real-life operating conditions. However, the detection accuracy significantly decreases for Level 0 and 1. Therefore, by applying the self-aware approach we can benefit from the low energy consumption of Level 0 and 1 by using them only when the detection confidence is good enough, otherwise, we use Level 2.

\section{Performance of our system}

Although Table $\mathrm{X}$ gives an estimation of the theoretical battery lifetime for each level, our system continuously switches between the three different energy scalable levels. Hence, a more realistic battery lifetime estimation is given in this subsection. We provide the results of a self-aware online cognitive workload detection setup. Based on our test sets, which are composed of 188 minutes collected from 24 subjects, our system runs 114 minutes in Level 0, 47 minutes in Level 1, and 27 minutes in Level 2. Table XI reports the probability of using each level based on a given confidence threshold calculated on the training set and the probable average current and energy consumption per level and for the self-aware operation.
TABLE XI: Performance of our system on the test set.

\begin{tabular}{lcccc}
\hline Self-aware Manager & L0 & L1 & L2 & Self-Aware \\
\hline Level's use Prob. (\%) & 60.64 & 25.00 & 14.36 & - \\
Confidence Threshold & 0.8 & 0.3 & - & - \\
Average Current* (mA) & 9.93 & 5.03 & 4.03 & 19.0 \\
Energy* $(\mu \mathrm{Ah})$ & 154.6 & 78.44 & 62.67 & 295.7 \\
\hline Detection Accuracy (\%) & & & & 79.25 \\
Battery life* -420mAh- (h) & & & & $\mathbf{2 2 . 1 1}$ \\
\hline * based on the level usage probability & & &
\end{tabular}

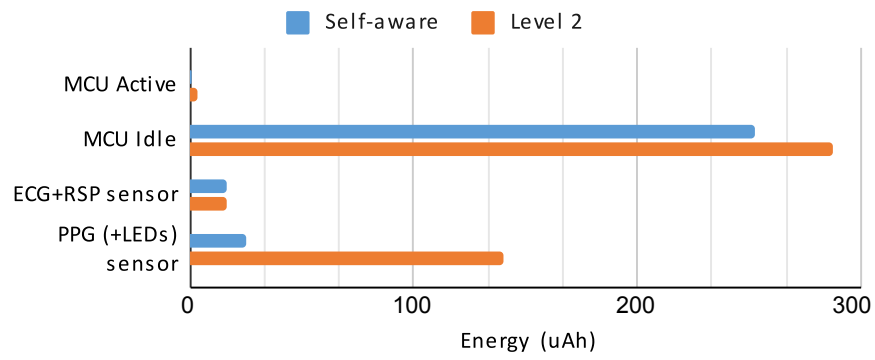

Fig. 7: MBioTracker's energy consumption per module on Level 2, and Self-aware operation for the test set data.

Furthermore, we report in Table XI the classification accuracy on our test set and the estimated battery lifetime. We conclude that, thanks to our self-aware approach, the battery lifetime is increased from 14.58 to 22.11 hours (for a case of a LiPo $420 \mathrm{mAh}$ battery) with a negligible classification accuracy degradation of $1.07 \%$, thus representing a $51.1 \%$ battery lifetime improvement. Fig. 7 provides an overview of our system energy breakdown for both operation modes Level 2 and Self-aware on the test set data. It clearly shows the energy consumption benefit of the self-awareness approach being more prominent for the PPG sensor and the idle time of the MCU.

Finally, when comparing our MBioTracker with state-of-the-art solutions for cognitive workload detection using wearable sensors, only our proposal includes both the required level of online multibiosignal monitoring and on-device processing, as highlighted in Table XII In this context, our use of edge computing together with the self-awareness approach allows reaching the highest autonomy level against data streaming solutions. Furthermore, our detection performance results are in a similar range to previous works despite our signal resolutions and processing constraints.

\section{CONCLUSION}

This paper presents MBioTracker, a new multimodal and wearable embedded system for online cognitive workload monitoring. The hardware of our system is a platform capable of real-time multichannel physiological signals acquisition and low-power processing. The software side of our wearable embedded system includes novel energy-aware bio-signal processing and applies the system selfawareness concept to enable scalable energy operation modes.

Our results demonstrate that MBioTracker can detect low and high cognitive workload levels during the test scenario of simulated search and rescue mission with drones. We have achieved an accuracy of $76.6 \%$ versus $79.3 \%$ in offline and a non-embedded system approach, respectively. Besides, when simulating the self-aware approach, we increase $51 \%$ the battery lifetime by switching between different energy scalable modes.

Finally, although we have designed MBioTracker specifically for cognitive workload monitoring, its adaptive new multi-channel physiological signals acquisition hardware platform, together with the implemented energy-aware modular signal processing architecture, makes it suitable to target other multimodal bio-monitoring applications in the near future. 
TABLE XII: Comparison between the proposed and state-of-the-art solution for cognitive workload detection applications.

\begin{tabular}{lllll}
\hline Ref. & Platform (size \& autonomy) & Data Processing & ML Solution & Detection Performance \\
\hline$[12]$ & $\begin{array}{l}\text { Shimmer }(65 \times 33 \times 12 \mathrm{~mm}, 16 \mathrm{~h}) \\
\text { E4 wristband }(44 \times 40 \times 16 \mathrm{~mm}, 24 \mathrm{~h})\end{array}$ & Data streaming, Offline & 10 Feat., RF model, & Acc: $84.1 \%$ \\
\hline$[13]$ & Simulated Platform (-) & Data streaming, Offline & 13 Feat., RF model, Self-aware (2-levels) & Gmean: $81.7 \%$ \\
\hline$[22]$ & $\begin{array}{l}\text { BioNomadix system } \\
(3 \text { modules: 60x40x20mm, 12h) }\end{array}$ & Data Logging, Offline & 24 Feat., XGBoost model & Acc: $80.2 \%$, Gmean: $75.54 \%$ \\
\hline This work & MBioTracker(62x52x9mm, 22h) & Online, edge-computing & 22 Feat., SVM model, Self-aware (3-levels) & Acc: $79.31 \%$, Gmean: $79.36 \%$ \\
\hline
\end{tabular}

\section{REFERENCES}

[1] K. H. Teigen, "Yerkes-Dodson: A Law for all Seasons," Theory \& Psychology, vol. 4, no. 4, pp. 525-547, 111994.

[2] A. Marinescu et al., "Exploring the relationship between mental workload, variation in performance and physiological parameters," IFACPapersOnLine, vol. 49, no. 19, pp. 591-596, 2016.

[3] B. Cain, "A Review of the Mental Workload Literature. Toronto," Defence Research and Development Canada, no. 1998, 2007.

[4] M. Ranchet et al., "Cognitive workload across the spectrum of cognitive impairments: A systematic review of physiological measures," Neuroscience \& Biobehavioral Reviews, vol. 80, pp. 516-537, 2017.

[5] J. Heard, C. E. Harriott, and J. A. Adams, "A Survey of Workload Assessment Algorithms," IEEE Transactions on Human-Machine Systems, pp. $434-451,2018$.

[6] F. Dell'Agnola, "Wearable and Self-Aware Machine Learning System for Online Cognitive Workload Monitoring and Drone Control," Ph.D. dissertation, Ecole Polythecnique Federal de Lausanne, 2020.

[7] A. Arza et al., "Measuring acute stress response through physiological signals: towards a quantitative assessment of stress," Medical and Biological Engineering and Computing, pp. 1-17, 8 2018. Available: http://link.springer.com/10.1007/s11517-018-1879-z

[8] N. Momeni et al., "Real-Time Cognitive Workload Monitoring Based on Machine Learning Using Physiological Signals in Rescue Missions," EPFL, Tech. Rep., 42019.

[9] L.-1. lan Chen et al., "Detecting driving stress in physiological signals based on multimodal feature analysis and kernel classifiers," Expert Systems with Applications, vol. 85, pp. 279-291, 112017.

[10] D. Giakoumis, D. Tzovaras, and G. Hassapis, "Subject-dependent biosignal features for increased accuracy in psychological stress detection," International Journal of Human-Computer Studies, vol. 71, no. 4, pp. 425-439, 2013.

[11] M. Gjoreski et al., "Monitoring stress with a wrist device using context," Journal of Biomedical Informatics, vol. 73, pp. 159-170, 92017.

[12] V. Montesinos et al., "Multi-modal acute stress recognition using offthe-shelf wearable devices," in 2019 41th Conference of the IEEE Engineering in Medicine and Biology Society (EMBC), July 2019.

[13] G. Masinelli et al., "Self-Aware Machine Learning for Multimodal Workload Monitoring During Manual Labor on Edge Wearable Sensors," IEEE Design Test, vol. 2356, no. c, p. 1, 2020.

[14] F. Forooghifar et al., "A Self-Aware Epilepsy Monitoring System for Real-Time Epileptic Seizure Detection," Mobile Networks and Applications, pp. 1-14, 82019.

[15] D. P. N TaheriNejad, A Jantsch, "Comprehensive observation and its role in self-awareness," Self, vol. 11, p. 1, 2016.

[16] A. Anzanpour et al., "Self-awareness in remote health monitoring systems using wearable electronics," in Design, Automation Test in Europe Conference Exhibition (DATE), 2017, 2017, pp. 1056-1061.

[17] S. Kounev et al., Self-aware computing systems. Springer, 2017.

[18] S. Betti et al., "Evaluation of an Integrated System of Wearable Physiological Sensors for Stress Monitoring in Working Environments by Using Biological Markers," IEEE Transactions on Biomedical Engineering, vol. 65 , no. 8, pp. 1748-1758, 82018.

[19] V. Kartsch et al., "BioWolf: A Sub-10-mW 8-Channel Advanced BrainComputer Interface Platform with a Nine-Core Processor and BLE Connectivity," IEEE Transactions on Biomedical Circuits and Systems, vol. 13, no. 5, pp. 893-906, 102019.

[20] S. Song et al., "A $769 \mu \mathrm{W}$ Battery-Powered Single-Chip SoC With BLE for Multi-Modal Vital Sign Monitoring Health Patches," IEEE Transactions on Biomedical Circuits and Systems, vol. 13, no. 6, pp. 1506-1517, 122019.

[21] R. Zanetti, A. Aminifar, and D. Atienza, "Robust Epileptic Seizure Detection on Wearable Systems with Reduced False-Alarm Rate," in
2020 42nd Annual International Conference of the IEEE Engineering in Medicine \& Biology Society (EMBC). IEEE, 7 2020, pp. 4248-4251.

[22] F. Dell'Agnola et al., "Cognitive workload monitoring in virtual reality based rescue missions with drones," in 12th International Conference on Virtual, Augmented and Mixed Reality, Copenhagen, Denmark, 72020.

[23] K. Lee et al., "A Multi-Functional Physiological Hybrid-Sensing E-Skin Integrated Interface for Wearable IoT Applications," IEEE transactions on biomedical circuits and systems, vol. 13, no. 6, pp. 1535-1544, 12 2019.

[24] "Empatica." Available: https://www.empatica.com

[25] "Biovotion AG." Available: http://www.biovotion.com/everion/

[26] "Zephyr ${ }^{\mathrm{TM}}$ Performance Systems - Performance Monitoring Technology." Available: https://www.zephyranywhere.com/

[27] "smartcardia," 2015. Available: http://www.smartcardia.com/

[28] M. Konijnenburg et al., "22.1 A $769 \mu \mathrm{W}$ Battery-Powered Single-Chip SoC with BLE for Multi-Modal Vital Sign Health Patches," in Digest of Technical Papers - IEEE International Solid-State Circuits Conference, vol. 2019-Febru. IEEE, 2 2019, pp. 360-362.

[29] Y.-S. Shu et al., "26.1 A 4.5mm 2 Multimodal Biosensing SoC for PPG, ECG, BIOZ and GSR Acquisition in Consumer Wearable Devices," in 2020 IEEE International Solid- State Circuits Conference - (ISSCC), vol. 2020-Febru. IEEE, 2 2020, pp. 400-402.

[30] S. Shah et al., "A Real-Time Vital-Sign Monitoring in the Physical Domain on a Mixed-Signal Reconfigurable Platform," IEEE transactions on biomedical circuits and systems, vol. 13, no. 6, pp. 1690-1699, 12 2019.

[31] S. Song et al., "A $769 \mu \mathrm{W}$ Battery-Powered Single-Chip SoC With BLE for Multi-Modal Vital Sign Monitoring Health Patches," IEEE Transactions on Biomedical Circuits and Systems, vol. 13, no. 6, pp. 1506-1517, 122019.

[32] S. G. Hart and L. E. Staveland, "Development of NASA-TLX (Task Load Index): Results of Empirical and Theoretical Research," in Human Mental Workload, P. A. Hancock and N. Meshkati, Ed. Amsterdam: North Holland Press., 1988, pp. 139-183.

[33] L. Orlandic et al., "REWARD: Design, Optimization, and Evaluation of a Real-Time Relative-Energy Wearable R-Peak Detection Algorithm *," in 2019 41st Annu Int Conf IEEE Eng Med Biol Soc. IEEE, jul 2019, pp. 3341-3347.

[34] P. Grossman and E. W. Taylor, "Toward understanding respiratory sinus arrhythmia: Relations to cardiac vagal tone, evolution and biobehavioral functions," Biological Psychology, vol. 74, no. 2, pp. 263-285, Feb 2007.

[35] Task Force of the European Society of Cardiology and the North American Society of Pacing and Electrophysiology., "Heart rate variability: standards of measurement, physiological interpretation and clinical use," Circulation, vol. 93, no. 5, pp. 1043-1065, 31996.

[36] P. R. Lewis et al., "A survey of self-awareness and its application in computing systems," in Fifth IEEE Conference on Self-Adaptive and Self-Organizing Systems Workshops. IEEE, 2011, pp. 102-107.

[37] S. Kounev et al., Self-Aware Computing Systems, 1st ed. Springer International Publishing, 2017.

[38] "STM32L151." Available: https://www.st.com/ microcontrollers-microprocessors/stm321151rd.html

[39] "nRF8001." Available: https://infocenter.nordicsemi.com/topic/struct nrf8000/struct/nrf8001_ps.html

[40] "AFE4300." Available: http://www.ti.com/lit/gpn/AFE4300

[41] "Lmt70." Available: https://www.ti.com/lit/gpn/lmt70

[42] “AFE4490." Available: http://www.ti.com/lit/gpn/afe4490

[43] "ADS1292R." Available: http://www.ti.com/lit/gpn/ADS1292R

[44] "Biopac MP160 Data Acquisition Systems." Available: https://www. biopac.com/product/mp150-data-acquisition-systems

[45] C. Orphanidou, "Signal Quality Assessment in Physiological Monitoring: Requirements, Practices and Future Directions." Springer, Cham, 2018, pp. 1-14. 


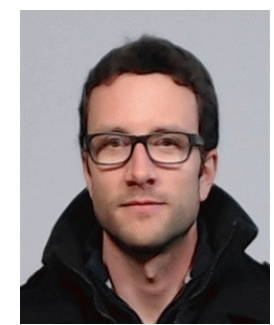

Fabio Dell'Agnola (M'18) received his $\mathrm{PhD}$ degree in electrical engineering from the Swiss Federal Institute of Technology Lausanne (EPFL) in 2020. During his PhD, he worked at the Embedded System Laboratory (ESL) from EPFL on a project aimed to design a wearable and self-aware machine-learning system for cognitive workload monitoring and drone control. Dr. Dell'Agnola is currently working in the $R \& D$ group at ABB in Quartino, Switzerland.

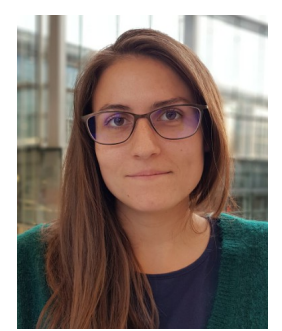

Una Pale received her BSc and MSc degrees in electrical engineering from the University of Zagreb, Croatia in 2014 and 2016, respectively. During an exchange at the Technical University of Vienna she decided to direct her research interest to biomedical applications. She worked for almost two years as a research assistant in the Clinical Neuroengineering Laboratory at the Swiss Institute of Technology Lausanne (EPFL), Switzerland. Currently she is pursuing her PhD in the Embedded Systems Laboratory at EPFL. Her research interests are mainly in biomedical signal processing, machine learning for health related applications, and design and application of hyperdimensional computing algorithms.

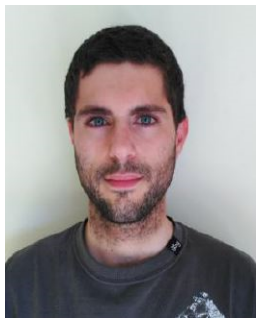

Rodrigo Marino received the BSc degree in Industrial Electronics Engineering and Automation from Universidade de Vigo, Spain, in 2015, and the MSc in Industrial Engineering in 2017 from the same University. He is currently working toward the Ph.D. degree in Industrial Electronics at Universidad Politécnica de Madrid. His current research area is in the field of machine learning applied to embedded systems, also known as expert embedded computing. He is involved in Spanish R\&D projects related to the enhance the acquisition systems, combining machine learning techniques in embedded systems to develop expert sensors for multiple fields. Furthermore, he has also participated in innovation projects to transfer the state-of-the-art expert systems to the industrial companies.

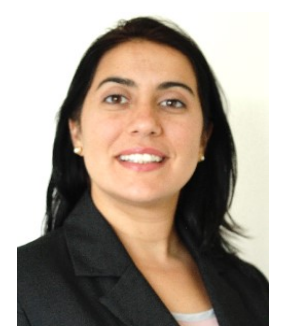

Adriana Arza Valdés is a Post-Doctoral researcher in the Embedded Systems Laboratory at the the Swiss Federal Institute of Technology Lausanne, Switzerland. She received her $\mathrm{PhD}$ degree in Microelectronic and Electronic Systems in 2017 and M.Sc. degree in Micro and Nanoelectronics Engineering in 2012, both from Autonomous University of Barcelona. Her research interests include energy-efficient algorithms and machine learning for edge health and wellness applications and analysis and interpretation of multimodal physiological data.

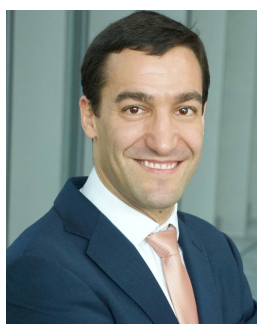

David Atienza (M'05-SM'13-F'16) is associate professor of electrical and computer engineering, and director of the Embedded Systems Laboratory (ESL) at the Swiss Federal Institute of Technology Lausanne (EPFL), Switzerland. He received his $\mathrm{PhD}$ in computer science and engineering from UCM, Spain, and IMEC, Belgium, in 2005. His research interests include system-level design methodologies for high-performance multi-processor systemon-chip (MPSoC) and low-power Internet-ofThings (loT) systems, including new 2-D/3-D thermal-aware design for MPSoCs and many-core servers, ultra-low power edge Al architectures for wireless body sensor nodes and smart consumer devices. He is a coauthor of more than 300 papers in peer-reviewed international journals and conferences, several book chapters, and seven patents. Dr. Atienza received the DAC Under-40 Innovators Award in 2018, IEEE TCCPS Mid-Career Award in 2018, an ERC Consolidator Grant in 2016, the IEEE CEDA Early Career Award in 2013, the ACM SIGDA Outstanding New Faculty Award in 2012, and a Faculty Award from Sun Labs at Oracle in 2011. He served as DATE 2015 Program Chair and DATE 2017 General Chair. He is an ACM Distinguished Member, an IEEE Fellow, and has served as IEEE CEDA President (period 2019-2020). 